\title{
LOS ESTÁNDARES EN INVESTIGACIÓN Y PUBLICACIÓN EN EL PROCESO DE LICENCIAMIENTO
}

\author{
The standards in research and publication in the \\ licensing process
}

Jorge Ybaseta - Medina ${ }^{1, a}$, Carmen Vera - Cáceres ${ }^{1, b}$.

1. Universidad Nacional San Luis Gonzaga de Ica, Perú.

a. Director de la Revista Médica Panacea.

b. Directora de la Unidad de Investigación de la Facultad de Medicina Humana.

En estos momentos se están desplegando todos los esfuerzos necesarios para mejorar nuestro papel como Universidad en lo relacionado: a la promoción ciudadana, en mejorar las competencias para el desarrollo del empleo, infraestructura adecuada, planes de investigación y objetivos académicos, no dejándose de mencionar que el trabajo de acreditación realizado nos está ayudando a cumplir parte de los requisitos para el licenciamiento puesto que ambas son complementarias para el efecto de la calidad universitaria (1).

En este proceso de adecuación a los lineamientos de evaluación de calidad de la educación superior de la Superintendencia Nacional de Educación Superior Universitaria (SUNEDU), en lo referente a la investigación, está claro que el cumplimiento de varios de estos indicadores son conducentes al licenciamiento de una universidad guardando estrecha relación con producción científica entre estos : investigadores calificados por CONCYTEC, publicaciones científicas indizadas, repositorio institucional integrado en el portal del Acceso libre a Información Científica para la Innovación (ALICIA), entre otros (2).

El Comité Editorial de la Revista Médica Panacea (RMP) sigue trabajando en lo que compete en la medida de los recursos y posibilidades, primero se trabajó algunos indicadores para la acreditación de la Facultad de Medicina Humana y actualmente nuestro proceso está orientado en una labor de apoyo a una mayor producción científica y publicación de temas que estén dentro de las líneas de investigación que se han priorizado, asimismo continuar con los cursos taller de redacción científica y lograr que los docentes puedan aspirar y tener publicaciones en base de datos como Medline o Scopus requisitos para ingresar al Registro Nacional de Investigadores en Ciencia y Tecnología de CONCYTEC.

En este sentido el Comité de Editores de la RMP continua haciendo esfuerzos para que los docentes y alumnos universitarios adquieran los conocimientos necesarios en la redacción de artículos de investigación como expresión de nuestros ánimos de un mayor compromiso en lo que se refiere la publicaciones científicas, iniciando este proyecto difícil que involucra a todos los que trabajamos en la Universidad, estamos por subir al primer peldaño se está a la espera de la evaluación para que la RMP alcance el registro en LATINDEX y sea integrada al portal de ALICIA del CONCYTEC al igual que el repositorio de la Universidad, estas plataformas virtuales que estamos iniciando a manejar cada vez más ayudan a conseguir indicadores preliminares y permiten la difusión de la investigación como la reutilización de los contenidos sobre todo para la realización de tesis y artículos originales a nivel nacional e internacional como viene ocurriendo actualmente (3).

Necesitamos que todas las autoridades, docentes y alumnos se involucren para que nuestras investigaciones continúen mejorando en calidad, hace 6 años que las tesis para optar el Título de 
Médico Cirujano luego de la contribución realizada por asesores y jurados están siendo transformadas en artículos originales por los autores seguido de revisión posterior de los editores y revisores externos resultando una investigación de mayor calidad que se publica en la RMP, que bajo una licencia Creative Commons versión 4.0 la misma que permite fortalecer el acceso, el intercambio o compartir el contenido científico en un mundo globalizado y en distintas legislaciones internacionales (4).

Como contribución cuando las investigaciones no son publicables los detalles de estas son informadas a la unidad de investigación para las gestiones de mejora.

\section{REFERENCIA BIBLIOGRÁFICAS}

1. Superintendencia Nacional de Educación Superior Universitaria. El modelo de licenciamiento y su implementación en el Sistema de abril Universitario Peruano 2015 . [ fecha de acceso disponible 07 de abril de 2018 ]. URL disponible en:

https://www.sunedu.gob.pe/direccion-de-licenci amiento/condiciones-basicas-de- calidad/

2. Alhuay Quispe J. Evaluación de investigación en universidades peruanas. En: Ponencia presentada en el centro de investigación, Perú: Universidad San Ignacio de Loyola; 2017. [Fecha de acceso disponible 07 de abril de 2018]. URL disponible en:

http://repositorio.usil.edu.pe/handle/USIL/2839
3. Gema Bueno de la Fuente. Modelo de repositorio institucional de contenido educativo (RICE): la gestión de materiales digitales de docencia y aprendizaje en la biblioteca universitaria. (Tesis Doctoral). Getafe: Departamento de biblioteconomía y documentación, Universidad Carlos III de Madrid; 2010. [ fecha de acceso disponible 07 de abril de 2018]. URL disponible en:

https://e-archivo.uc3m.es/bitstream/handle/100 16/9154/Tesis\%20doctoral-;jsessionid=3F65A28 49628C20E94AE80703C01D41B? sequence $=1$

4. Cardona A. Copileft y las licencias creative commos en la industria editorial. Universidad de Antioquia. Escuela Interamericana de Bibliotecología Editorial Universidad de Antioquia Medellín; junio de 2010. [fecha de acceso disponible 09 de abril de 2018 ]. URL disponible en:

http://bibliotecadigital.udea.edu.co/bitstream/1 0495/1452/1/Monograf\%C3\%ADa\%20Angela\%2 0\%C3\%BAltima\%20version.pdf 